\title{
Characterization and optical properties of organic dye films as recording media
}

\author{
V.G. Kravets, K.L. Vinnichenko*, O.V. Prygun \\ Institute for Information Recording Problems, NASU, Kyiv, Ukraine \\ *Department of Physics, Kyiv Taras Shevchenko University, Kyiv, Ukraine
}

\begin{abstract}
Efficiency of medium choice for optical information recording is generally determined by homogeneity degree of material on the recording surface and by its structure on the film depth. In this respect pyrosolynes should be the prospective materials. Pyrosolyne dye films were produced on the glass substrates initially remained at room temperature $T=20^{\circ} \mathrm{C}$ by using different techniques such as vapor deposition and sol-gel modified reactions. One of the precise control methods of material surface physical-chemical state is ellipsometric method. In addition, the optical transmission measurements were carried out for these films within the wavelength range $\lambda=200-630 \mathrm{~nm}$ at two different polarization ( $p$ - and $s$-) of the incident light. It has been shown that the absorption bands near $n=1300 \mathrm{~cm}^{-1}$ are present in ellipsometric parameters spectra in case of the vapor deposited dye film samples. The transmission optical measurements of organic films in the visible range without polarizer showed presence of the absorption bands near $\lambda=300$ and $520 \mathrm{~nm}$. The first absorption band was much more intensive than another. It has been determined that the transmission values measured in polarized in $p$ and $s$-directions light differs by $5-8 \%$ in comparison with data measured without polarizer.
\end{abstract}

Keywords: dye film, information recording, vacuum evaporation process, spin-coating method, IR- absorption.

Paper received 24.07.00; revised manuscript received 05.09.00; accepted for publication 12.12.00.

\section{Introduction}

The predominant compact disk $(\mathrm{CD})$ mastering processes are based on either photoresist or dye-polymer recording media. There are several advantages of using the dye-polymer mastering process over that of photoresist. The direct effect of recording on the dye medium allows to control the quality of the recording process in the manufacture of the CD or digital versatile disk (DVD) masters. Besides, the photoresist mastering process requires additional chemical developing. The dye recording is based on a thermal recording mechanism [1].

Efficiency of medium choice for optical information recording is generally determined by homogeneity degree of material on the recording surface and by its structure on the film depth. It is shown that pyrosolynes should be the prospective recording materials, because these dyes are highly homogeneous on surface and in depth.

In this paper, the basic optical characteristics of the vacuum evaporated disk in comparison with such characteristics of the disc fabricated by conventional spin-coating method are studied.
The main aim of the present work is to clarify the mechanism of dye layer absorbancy influence on formation of the small recording marks.

\section{Experimental}

The samples of dye recording media were prepared by thermal vacuum evaporation at the background pressure of about $10^{-3} \mathrm{~Pa}$ on glass disks $160 \mathrm{~mm}$ in diameter and $2.1 \mathrm{~mm}$ in thickness in the commercially available vacuum chamber having resistive thermal evaporators. These glass substrates were also coated with a dissolved dye in Si-polymer organic film by spin-coating. The thickness of the film layers was approximately $0.15 \mu \mathrm{m}$.

The transmission spectra were recorded by SF-20 double-beam spectrophotometer in the region $0.2<\lambda<10 \mu \mathrm{m}$. The sample thicknesses were measured by means of optical multiple beams interference method and multiple angle ellipsometry. The layers have been characterized by infrared spectroellipsometry using a Fourier-transform spectrometer in the middle infrared range (wavenumber value range $v=400-5000 \mathrm{~cm}^{-1}$ ) in accordance with the 


\section{V.G. Kravets et al.: Characterization and optical properties of organic ...}

scheme of polarimetric Beattie-Conn method [2,3]. Transmission electron and atomic force microscopy probed film morphology and structure.

\section{Results and discussion}

Integral absorption spectra of the vacuum evaporated and spin-coated dye films are characterized by presence of a broad intensive band at $\lambda=290 \mathrm{~nm}$ and a weak band near $500-520 \mathrm{~nm}$ (Fig. 1). The peak in the near UV region $(290 \mathrm{~nm})$ is due to the band-to-band transition in pyrosolyne organic dyes since we deal with the wide band-gap. The peak at about $510 \mathrm{~nm}$ in the transmission spectrum could be associated with intercenter transition of organic complex. The similar peak was observed for original organic dye powder [4]. This experimental fact confirms that the structure of organic dye was not destroyed during the film deposition in the abovementioned technology.

Fig. 2 shows the IR transmission spectra of the dye recording layers. For the dye film distinct IR-transmission minimum occurs at $v=1350 \mathrm{~cm}^{-1}$ is observed. The organic dye optical properties were also studied by means of spectroellipsometric method realised in the BeattieConn scheme using Fourier transform spectrometer in the IR within the wave number value range $v=500-5000 \mathrm{~cm}^{-1}$. The optical characteristics $\tan \Psi$ and $\cos \Delta$ vs wavelength were determined [3]. The IR-ellipsometric measurements using a Fourier-transform spectrometer also demonstrated existence of the perceptible singularity at the same wavenumber value $1350 \mathrm{~cm}^{-1}$. Pyrosolyne organic complex bonds have $\mathrm{sp}^{2}$ configuration. The pyrosolyne lattice has two $\sigma$-bonds, one $p \pi$-bond, and it has two electrons in a filled valence band [4,5]. Pyrosolyne ring has $\mathrm{C}=\mathrm{C}$ and $\mathrm{C}=\mathrm{N}$ in-plane vibrations in the two band range, one from 1300 to $1500 \mathrm{~cm}^{-1}$ and the other from 1150 to $2500 \mathrm{~cm}^{-1}$. The absorption band occurred for pyrosolynes in the region $v=1250 \div 1580 \mathrm{~cm}^{-1}$ is due to the $\mathrm{C}=\mathrm{C}$ and $\mathrm{C}=\mathrm{N}$ stretching vibrations. The $\mathrm{C}=\mathrm{N}$ band for tetrahedral

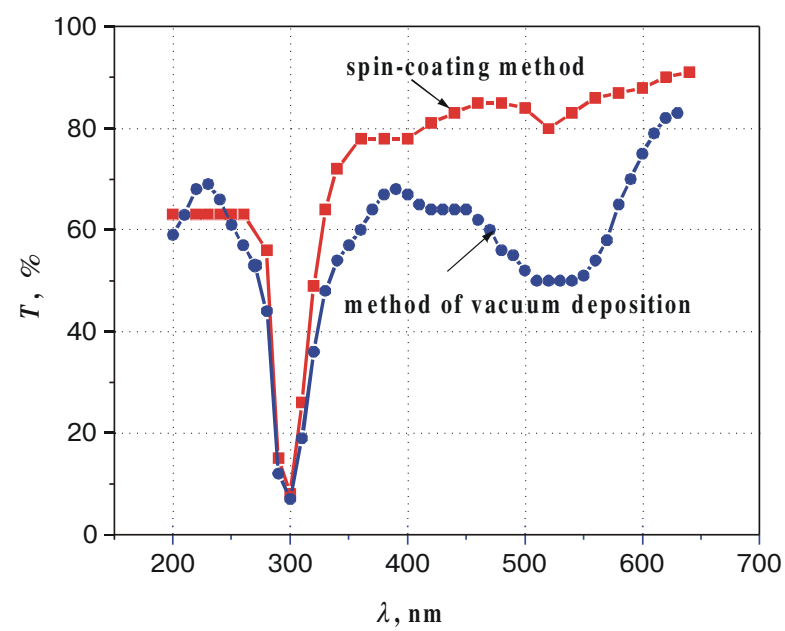

Fig. 1. Transmission spectra of pyrosolyne dye film at different regimes.

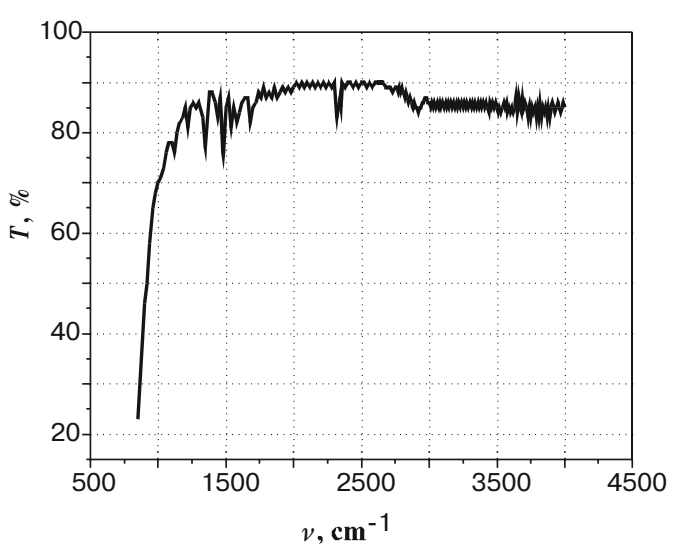

Fig. 2. IR transmission spectrum of pyrosolyne dye film.

aromatic amines is found in $n=1300-1380 \mathrm{~cm}^{-1}$ range. The IR spectra exhibit a strong absorption band at $800 \mathrm{~cm}^{-1}$ which is ascribed to Si-C bonds in the pyrosolyne organic complex.

These dye films have two absorption bands, however the absorption band near $300 \mathrm{~nm}$ is more appropriate for information recording in DVD-R technology. It was shown that organic disc exhibits excellent recording performance for small marks less than $0.5 \mu \mathrm{m}$ in length. The absorption band for vacuum deposited dye films at $510 \mathrm{~nm}$ is more intensive than for spin-coated film (Fig. 1). That is why, it could be inferred that the vacuum evaporated films possess a larger recording sensitivity in comparison with dye-polymer layer. The power margin of the evaporated disk is much wide than that of the spin-coated disk. The evaporated disk is advantageous for high density recording. In dye recording media the hole formation due to the sublimation of dye material is still origin of the decrease of the refractive index, and this mechanism leads to increase of the refractive index changes at the recorded pits. During the recording process laser energy destroys the bonds between $\mathrm{C}$ and C, N, Si atoms.

The results of the spectroscopic measurements of photoinduced dichroism in dye films are presented in Fig. 3. Parallel and perpendicular absorption spectra components $\left(\alpha_{p}\right.$ and $\left.\alpha_{s}\right)$ were measured $v s$ linearly polarized excitation light. One can observe (Fig. 3) that the absorption index $\alpha_{p}$ for light polarized parallel to the incident plane becomes smaller than for light polarized perpendicular to this plane. Perhaps, these dye films provide high reproducibility of photoorientation transformation under irradiation by light with orthogonal polarization. Analysis of the $\tan \Psi(\lambda)$ dependencies showed that $\tan \Psi(\lambda)$ values exceed unity for the absorption band, it has permitted to conclude that the film structure is anisotropical. The intensity of light consecutively passed through polarizer and an anisotropically absorbing dye film is given by the relationship [6]:

$I_{\text {out }}=\frac{I_{\text {max }}-I_{\min }}{2}-\frac{I_{\max }-I_{\min }}{2} \cos (2 \Theta)$ 


\section{V.G. Kravets et al.: Characterization and optical properties of organic ...}

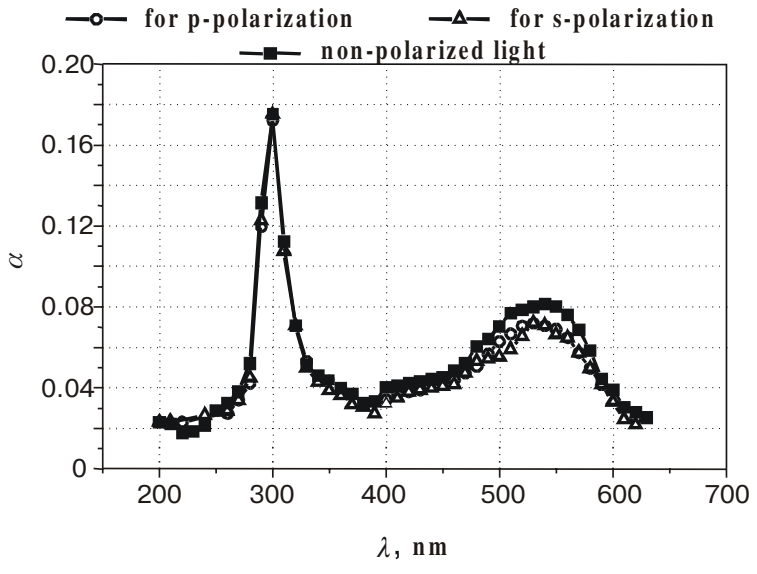

Fig. 3. Absorption spectra for dye films at different polarizations

where angle $\Theta$ is the orientation (in the plane of the sample surface) of the absorbing dipole with respect to molecule fast axes. The minimum intensity of light measured during the modulation cycle is $I_{\min }$, while $I_{\max }$ is the maximum. Thus, the average amplitude of intensity modulation can be given as:

$R=\frac{I_{\max }-I_{\min }}{I_{\max }+I_{\min }}$

With the assumption that Beer's law remains valid for anisotropically absorbing sample, the anisotropic extinction coefficient can be obtained from $R$-value:

$\varepsilon=-\frac{1}{h c} \log \left(\frac{1-R}{1+R}\right)$

where $\varepsilon$ is a molar extinction coefficient in $\mathrm{mol}^{-1} \mathrm{~cm}^{-1}, h$ is the film thickness and $c$ is aconcentration of an absorbing substance in the sample. Considering that the number of absorbing centers is different for vacuum deposited dye films in comparison with spin-coated films, one could explain the observed distinctions between spectra in parallel and perpendicular polarized light.
From the structure measurements it was found that anisotropic grains (clusters) existing in dye films small (about $1 \div 2 \mathrm{~nm}$ ). When the optical axes of the grains (fast or slow) are directed along the polarization vector of the exciting light, we can obtain some minimum or maximum of intensity in the light passed through polarizer and film. In this work it was shown that those dye molecules that are oriented perpendicular to the plane of light polarization absorb light more intensively.

\section{Conclusions}

1. It is shown that pyrosolyne dye films can be used as recording media in optical compact disks because the structure of the dye is not destroyed after the deposition process.

2. The vacuum evaporation process in comparison with spin-coating technique is more appropriate for recording film deposition because it provides media with higher absorption index in the region of information recording $(200-600 \mathrm{~nm})$. The power margin of the evaporated disk is much wider than that of the spin-coated disk.

3. The dye films have disordered structure and demonstrate anisotropic optical properties, which strongly influence on recording process.

\section{References}

1. I.H. Rilum. Digital versatile disk mastering using dye-polymer media // Proc. SPIE «Optical Storage» (ISOS'96), 2931, p.148-153, (1996).

2. A. Roseler, Infrared Spectroscopic Ellipsometry, AcademieVerlag, Berlin, 1990, $160 \mathrm{p}$.

3. A. Roseler, E. Korte, L.V. Poperenko, V.G. Kravets, E.L. Vinnichenko, Optical properties of Organic Films for Compact Disk // Mol. Cryst. Liq. Cryst., 324, p. 37-44, (1998).

4. M. Pope and C.E. Swenberb, Electronic Processes in Organic Crystals Clarendon Press, Oxford University Press, New York, 1982.

5. G. Socrates, Infrared Characteristic Group Frequencies, Sec. Ed., JOHN WILEY \& SONS 1994, p.54-61

6. D.A. Higgins, D.A. Vanden Bout, I. Kerimo and P.F. Barbara. Polarization-modulation near-field scanning optical microscopy of mesostructured materials // J. Phys. Chem. 100, p.1379413803, (1996). 